\title{
Habilidades mentalistas en niños argentinos de 4 a 6 años: Adaptación de una escala de desarrollo de Teoría de la Mente
}

\section{Mentalizing skills in argentine children between the ages of four and six:}

\section{Adaptation of a development scale of Theory of Mind}

\author{
Maira Querejeta ${ }^{1}$, María Justina Romanazzi² y Julieta Fachal ${ }^{3}$
}

${ }^{1}$ Doctora en Psicología. Investigadora Adjunta en la Comisión de Investigaciones Científicas de la Provincia de Buenos Aires (CIC), Argentina. Profesora Adjunta de la Facultad de Humanidades y Ciencias de la Educación de la Universidad Nacional de La Plata (UNLP).

E-mail: mairaquerejeta@gmail.com

${ }^{2}$ Licenciada y Profesora en Psicología. Becaria Doctoral en la Comisión de Investigaciones Científicas de la Provincia de Buenos Aires (CIC), Argentina. Extensionista.

E-mail: mariajustina.r@gmail.com

${ }^{3}$ Licenciada y Profesora en Psicología. Becaria Doctoral en la Comisión de Investigaciones Científicas de la Provincia de Buenos Aires (CIC), Argentina. Extensionista.

Este trabajo fue subsidiado por la Comisión de Investigaciones Científicas de la Provincia de Buenos Aires (CIC), Argentina.

Centro de Estudios en Nutrición y Desarrollo Infantil (CEREN) de la Comisión de Investigaciones Científicas de la Provincia de Buenos Aires (CIC).

La Plata, Argentina.

\section{Resumen}

Este trabajo presenta los resultados del estudio sobre habilidades mentalistas en niños preescolares argentinos. Su interés reside en la importancia que revisten los logros conceptuales en el desarrollo de la Teoría de la Mente y el método adecuado para medirlos, dada la escasez de estudios sobre el tema en el ámbito local. Con un diseño descriptivo transeccional se examinaron 105 niños (4 a 6 años de edad) con la Escala de Teoría de la Mente de Wellman y Liu (2004), compuesta por 7 tareas diferenciadas, adaptada al español rioplatense. Entre los resultados de este estudio se destaca que, al igual que los hallazgos reportados por los autores de la escala, los porcentajes de respuestas correctas aumentan con la edad cronológica y disminuyen de acuerdo a la complejidad de la tarea. La secuencia y la dificultad creciente de las tareas son consistentes, aunque tal como lo proponen Wellman y Liu es necesario limitar las siete tareas originalmente propuestas a cinco, para ajustarse a los requerimientos de un escalograma. Los porcentajes de respuestas correctas hallados son menores a los encontrados en la investigación original teniendo en cuenta, además, que no se incluyeron niños de 3 años. La escala resulta un instrumento adecuado en la medida que proporciona evidencia empírica de la complejidad de las habilidades involucradas, así como de su progresión, imposibles de ser aprehendidas a través de una tarea única, tal como sucede en las evaluaciones que incluyen solo tareas de falsa creencia. 
Palabras clave: Teoría de la Mente; preescolares; evaluación psicológica; desarrollo infantil.

\section{Abstract}

This work introduces the results obtained in the study of mentalizing skills in pre-school Argentine children. Interest in this lies on the importance of conceptual achievements marking the normal development of the Theory of Mind (TM) and the appropriate way to measure them, and on the lack of studies on the subject in the region. The TM is understood as the capacity to infer emotions, beliefs and desires, and to use such inferences to predict and explain one's behavior as well as that of others. This theory has been studied thoroughly for the past 40 years due to its implications for communicative competence and social interaction, given that if a child fails to understand the relations between behavior and mental states, he or she will have difficulties understanding social situations in everyday life. Its assessment has changed based on how this theory has been conceptualized in connection with its birth and development. The initial conception, which considered it as a single capacity acquired once and for all, was replaced by a more gradualist approach. This enabled the enlargement of the notion of TM to a series of increasingly elaborate and flexible conceptual acquisitions formed throughout a child's psychological development. Based on the hypothesis that a series of mentalist achievements following a predictable sequence occur during a child's normal development, Wellman and Liu (2004) designed a scale which operationalizes the TM. The instrument, which was to be used with pre-school children, consisted of 7 tasks which examine various skills: 1. Diverse desires, 2. Diverse beliefs, 3. Knowledge access, 4. Contents false belief, 5. Explicit false belief, 6. Belief-emotion and 7. Real-aparent emotion. The aim of this paper is to present the results obtained with Wellman and Liu's TM Scale in pre-school children living in the city of La Plata, Province of Buenos Aires (Argentina), and to compare them against the results obtained by the authors. With a cross-sectional descriptive design, 105 children aged between 4 and 6 were tested using the TM Scale adapted to Rioplatense (River Plate) Spanish. Wechsler Preschool and Primary Scale of Intelligence (WPPSI III) was also used in order to check intellectual level and exclude the protocols of the children who scored below 70 in the Total IQ. Among the results of this study, as well as the findings reported by the authors of the scale, it is noteworthy that the percentage of right answers increases with chronological age and decreases in relation to task complexity. The sequence and the increasing difficulty of the tasks are consistent, however, as proposed by Wellman \& Liu, it is necessary to limit the original 7 tasks to 5 , to conform with the requirements of a scalogram within the ages taken into consideration. The percentages of correct answers found in this study are lower than those found in the original investigation, taking into account, in addition, that 3 years old children were not included. The scale constitutes an adequate instrument in that it provides empirical proof of the complexity of the skills involved as well as of their progression, both of which are impossible to apprehend through a single task, as is the case of the assessments including only false belief tasks. All in all, the differences found in the performance of the participants included with respect to the original study require further research in not only cross-sectional but also longitudinal studies of clinical and non-clinical populations.

Keywords: Theory of Mind; pre-school children; psychological evaluation; child development

\section{Introducción}

La capacidad de atribuir estados mentales a sí mismo y a los demás constituye una de las 
competencias fundacionales de la cognición humana. Esta capacidad, conocida en la literatura especializada como Teoría de la Mente (TM) (Carpendale y Lewis, 2006; Dennett, 1978; Olson, 1988; Perner, 1988, 1994; Wimmer y Perner, 1983), no sólo consiste en inferir emociones, creencias y deseos, sino también en utilizar dichas inferencias para predecir y explicar los comportamientos propios o ajenos (Buitelaar, van der Wees, Swaab-Barneweld y van der Gaag, 1999).

La TM ha sido intensamente estudiada en los últimos 40 años (Carpendale y Lewis, 2006; Olson, 1988; Perner, 1988, 1994; Wimmer y Perner, 1983) por sus implicancias en la competencia comunicativa que se pone de manifiesto en la interacción social. Efectivamente, si un niño no comprende la intencionalidad y las relaciones entre la conducta y los estados mentales, tendrá dificultades para entender las situaciones sociales de la vida cotidiana y actuar en consecuencia (Astington y Jenkins, 1995; Puche Navarro, 2002).

El interés por el estudio de la TM se remonta a fines de la década del 70 a partir de los trabajos pioneros de Premack y Woodruff (1978)realizados con primates. El concepto ganó progresivamente visibilidad entre otros investigadores, que comenzaron a estudiar niños pequeños para intentar comprender cómo se adquiere esta capacidad (Vesterinen, 2008). Los primeros psicólogos en interesarse por este campo fueron Wimmer y Perner, quienes en 1983 diseñaron la tarea clásica de Falsa Creencia (FC), que tuvo como objetivo operacionalizar la competencia de los niños en la atribución de estados mentales.

La aplicación de diferentes tareas de FC en diversos estudios demostró que los niños menores de cuatro años suelen fallar al responder las preguntas que dichas tareas demandan (Astington, 1998; Astington y Barriault, 2001; Baron-Cohen, Leslie y Frith, 1985; Wellman, 1995; Wimmer y Perner, 1983). Esto llevó a algunos investigadores a sostener la ausencia de una TM antes de los cuatro años de edad.
Con el avance de las investigaciones sobre este tema, la idea de la TM como una capacidad unitaria que se adquiere de una vez y para siempre comenzó a reemplazarse por un enfoque más gradualista del concepto (De Rosnay y Hughes, 2006; Dunn, 1988; Wellman, 1993). Esto permitió ampliar la noción de TM más allá del paradigma de evaluación de la FC para pasar a considerarla una serie de adquisiciones conceptuales cada vez más elaboradas y flexibles que se constituyen a lo largo del desarrollo psicológico infantil.

La evaluación de la TM ha variado en función de cómo ha sido conceptualizada en relación con su emergencia y desarrollo (Benavides Delgado y Roncacio Moreno, 2009). Las tareas que involucran el paradigma de la FC se utilizaron a principios de la década de los 80, bajo el consenso de los investigadores en concebirla como un salto evolutivo que se expresa a través de su "comprensión entre los cuatro y los cinco años de edad" (Resches, Serrat, Rostan y Esteban, 2010, p. 316).

Posteriormente, como consecuencia del cambio de perspectiva, surgido en parte de la indagación de la TM en distintas poblaciones clínicas y no clínicas y en diferentes contextos sociales, se hizo necesario el diseño de nuevas tareas para evaluar el funcionamiento mentalista. Wellman y Liu (2004) elaboraron la Escala de Teoría de la Mente bajo la consideración de que "la TM engloba muchas comprensiones más allá de las falsas creencias" (Wellman, 2016, p. 6) y que se trata de una serie de adquisiciones conceptuales progresivamente más complejas que aparecen de manera gradual a lo largo del desarrollo.

\section{Escala de Teoría de la Mente de Wellman y Liu}

Esta escala fue diseñada sobre la hipótesis de que en el desarrollo normal del niño se produce una serie de logros en la TM, que siguen una secuencia predecible. Los autores 
se basan en los hallazgos de múltiples investigaciones, que han establecido que "en la edad preescolar es que los niños comprenden explícitamente algunos puntos clave sobre los deseos y las acciones intencionalesantesde comprender explícitamente las creencias" (Wellman, 2016, p. 6).

Consiguientemente, un instrumento que contemplara distintos niveles de dificultad podría llegar a operacionalizar la TM de manera más adecuada, tomando en cuenta estos dos tipos de estados mentales -las creencias y los deseos- para explicar cómo los seres humanos comprendemos la mente y actuamos en consecuencia (Bartsch y Wellman, 1995). Por un lado, las creencias aluden a aquellos estados mentales que intentan reflejar algo verdadero acerca del mundo. En ese sentido, incluyen estados mentales tales como conocimientos, opiniones, conjeturas, convicciones e intuiciones. Por otro lado, los deseos conforman una categoría que incluye estados mentales de intención [pro attitudes] hacia o acerca de algo. Según esta teoría psicológica, las acciones de las personas se basan en estos dos estados mentales. Es decir, estarían motivadas por la creencia de que realizando esa acción, podrían alcanzar lo que desean.

A partir del estudio de conversaciones infantiles acerca de la mente, los autores logran conceptualizar la progresión en el dominio de los estados mentales referidos a deseos y creencias, diferenciando tres fases en su desarrollo:

1) Psicología temprana de los deseos. Antes de los tres años de edad, los niños hablan de deseos, esencialmente de lo que quieren y les gusta en una variedad de situaciones: deseos de objetos, deseos de acciones, deseos de estados de cosas actuales o futuros, así como diferenciar sus propios deseos de los demás. Apelan a los deseos con el fin de explicar la acción, la emoción y la interacción humana.

Sin embargo, no comprenden ni hacen referencia a los pensamientos o creencias. Bartsch y Wellman (1995) plantean que esta etapa se explica por el hecho de que los deseos son estados mentales no representacionales, es decir, refieren directamente a objetos del mundo real. Mientras que las creencias son estados representacionales que exigen otro tipo de contenidos mentales, en tanto aluden a pensamientos sobre esos objetos.

2) Psicología intermedia de los deseos-creencias. A partir de los tres años, los niños comienzan a referirse a las creencias y a atribuirlas a las personas, aunque todavía recurren mayoritariamente a los deseos para explicar y razonar sobre las acciones. Conversan sobre pensamientos ficticios (ideas imaginarias o desconectadas del estado real de las cosas), sobre creencias (pensamientos de que algo es así) y sobre creencias falsas (pensamientos de que algo es así, cuando en realidad no lo es). Pero aún hablan de los deseos mucho más a menudo que de los pensamientos y las creencias.

3) Psicología de las creencias-deseos. Desde los cuatro años en adelante, los niños ponen a las creencias en el centro de la escena y recurren de manera sistemática a ellas para dar cuenta de las acciones. No sólo advierten que las personas tienen pensamientos y creencias, sino también que éstos son cruciales para explicar por qué hacen ciertas cosas; de este modo, las acciones dirigidas a la consecución de los deseos están inevitablemente formadas por sus creencias sobre el mundo. Las concepciones acerca del mundo (las cuales podrían ser erróneas) son las que determinan los planes y actos de las personas.

Para la construcción de la escala de TM, además de considerar estos hallazgos teóricos, Wellman y Liu (2004) realizaron un meta análisis de investigaciones en las que se comparaba la comprensión de diferentes estados mentales, con el objetivo de obtener una idea general acerca de cuáles resultan más fáciles de comprender en el período de la edad preescolar. 
Del estudio realizado concluyeron un orden tentativo de dificultad de los conceptos involucrados en el desarrollo de la TM, que sirvió como información preliminar para el diseño de la escala.

De este modo, elaboraron un instrumento destinado a niños en edad preescolar debido a los cambios decisivos en la comprensión de los estados mentales que se producen a esta edad. La escala quedó conformada por siete tareas que examinan distintas habilidades:

1) Deseos diferentes: Diferenciación del deseo propio con respecto del ajeno sobre un mismo objeto.

2) Creencias diferentes: Discriminación de creencias diferentes de la propia sobre un mismo objeto, sin conocer cuál es la verdadera o la falsa.

3) Acceso al conocimiento: Predicción del conocimiento que puede tener el otro sobre un objeto.

4) Falsa creencia de contenido: Atribución a otro de una falsa creencia acerca del contenido de un recipiente prototípico, una vez que se conoce el verdadero contenido de dicho recipiente, que difiere del usual.

5) Falsa creencia explícita: Reconocimiento de que otro puede tener una creencia distinta de la realidad que puede ser falsa.

6) Creencia-emoción: Predicción de una emoción a partir de una creencia falsa.

7) Emoción real-aparente: Comprensión de que una persona puede sentir una determinada emoción, pero expresar una diferente.

Estas tareas resultan comparables en cuanto al formato, material utilizado y tipo de respuestas que demandan por parte del niño. Esta característica permite que las mismas varíen en dificultad en función de las habilidades que examinan y no de otros aspectos relacionados. Las siete tareas se administran siguiendo un orden de dificultad creciente, siendo las primeras las que examinan aquellas habilidades que aparecen más tempranamente en el desarrollo.

Según el patrón de respuestas obtenido por Wellman y Liu (2004) en la administración de la escala a 75 niños de 3 a 6 años, sólo cinco de las siete tareas originalmente planteadas se ajustaron al modelo del escalograma propuesto por Guttman'1. Dos pruebas fueron eliminadas (Falsa creencia explícita y Creencia-emoción) porque no resultaban discriminativas de acuerdo a la edad, es decir no se observaban diferencias significativas entre un nivel y otro. Por otro lado, las cinco seleccionadas resultaron "más estrechamente comparables en cuanto a los soportes, los materiales y los formatos de las preguntas" (Wellman y Liu, 2004, p. 531).

En un trabajo posterior, Wellman (2016) menciona estudios realizados sobre más de 500 preescolares de distintos países (Estados Unidos, Canadá, Australia y Alemania) con esa batería de tareas, que dan por resultado un orden de dificultad claro y consistente (por ejemplo, Kristen, Thoermer, Hofer, Aschersleben y Sodian, 2006; Peterson, Wellman y Liu, 2005; Wellman y Liu, 2004). No obstante, Wellman (2016) sostiene que "dentro de la consistente trayectoria del desarrollo en la edad preescolar existen variaciones significativas entre países en cuanto al tiempo de estos logros. Las variaciones son evidentes no solo entre países, sino que también entre individuos" (p. 6). Para Liu, Wellman, Tardif y Sabbagh (2008) esas discrepancias en los tiempos de adquisición de las habilidades mentalistas son el resultado de múltiples factores socioculturales y lingüísticos que conjuntamente dan forma al desarrollo de la TM. Entre estos factores se identifican particularmente aquellos que se definen por formas específicamente culturales de comprender los estados mentales. Por ejemplo, Lu, Su y Whang (2008) encontraron que la cultura euroamericana hace énfasis en las vivencias individuales de los estados mentales mientras que las culturas orientales promueven la atención en la significación de las interacciones con los otros en los ${ }^{1}$ El procedimiento de Guttman se describe en el apartado de Resultados. 
estados mentales. Por otra parte, las diferencias individuales han sido analizadas y vinculadas a las capacidades lingüísticas (Milligan, Astington y Dack, 2007), a las habilidades del funcionamiento ejecutivo (Serrano Ortiz, 2012) y a la calidad de las interacciones en el contexto familiar (Dunn, Brown, Slomkowski, Tesla y Youngblade, 1991).

Asimismo, Wellman (2016) plantea la influencia de la TM sobre las relaciones infantiles en la edad escolar entre pares y profesores, así como con el aprendizaje. Sostiene que para "pensar sobre los niños y la escolaridad, es importante considerar posibles avances de la TM en niños mayores" (p. 8). Al respecto otros investigadores han desarrollado tareas que pueden evaluar avances posteriores en las habilidades mentalistas, tales como la comprensión del sarcasmo, la ironía y la metáfora en niños mayores e incluso en adultos (O'Reilly, Peterson y Wellman, 2014; Peterson, Wellman y Slaughter, 2012).

Wellman y Liu (2004) concluyen que su escala establece una progresión de logros conceptuales que refleja el ritmo de la comprensión de la TM en niños de desarrollo normal y además, constituye un método para evaluarlo (un método validado también con datos longitudinales; Wellman, Fang y Peterson, 2011).

\section{El presente trabajo}

El propósito de este trabajo es presentar los resultados obtenidos con la Escala de TM de Wellman y Liu (2004) en niños preescolares que residen en ciudad de La Plata, provincia de Buenos Aires (Argentina) y compararlos con los obtenidos en el estudio original.

Este estudio se justifica en la consideración de tres cuestiones. La primera, alude a la importancia que la TM tiene para el desarrollo psicológico infantil y a la escasez de estudios sobre el tema en el ámbito local. La segunda, refiere al valor de disponer de un método adecuado para evaluar con exactitud y de manera informativa el progreso de la comprensión social cognitiva en niños prees- colares. La tercera, a la relevancia de explorar las semejanzas o diferencias que podrían encontrarse en nuestro medio, en la complejidad creciente de las tareas propuestas para captar la secuencia del desarrollo de las habilidades mentalistas.

\section{Método}

\section{Participantes}

La muestra original estuvo compuesta por 130 niños y niñas de entre 4 y 6 años de edad. Se consideraron como criterios de inclusión la escolarización de los participantes de acuerdo a la edad cronológica y la semejanza en el nivel sociocultural de procedencia. Los criterios de exclusión fueron la presencia de dificultades específicas del lenguaje, discapacidad intelectual u otras patologías asociadas al desarrollo.

Respecto del nivel intelectual, se omitieron los casos con CI total menor a 70 (WPPSI III) $(\mathrm{n}=25)$, que presentaron un desempeño homogéneamente descendido. De este modo,en el grupo de niños finalmente incluidos en la muestra se obtuvieron las siguientes medidas descriptivas: $M=91.79, D E=11.29$, rango: 71 a 116.

La muestra final estuvo conformada por 105 sujetos de ambos géneros de tres grupos etarios, de 4, 5 y 6 años de edad. Específicamente, participaron 45 niños de 4 años $(\mathrm{M}=$ 4.4; rango: 4.0 a 4.10; 22 varones, 23 mujeres), 38 niños de 5 años $(\mathrm{M}=5.4$; rango: 5.0 a $5.10 ; 19$ varones, 19 mujeres) y 22 niños de 6 años $(\mathrm{M}=6.5$; rango: 6.3 a 6.6 ; 8 varones, 14 mujeres). Los niños asistían a establecimientos educativos estatales o privados $(\mathrm{n}=$ 8) de Gran La Plata. De sus legajos escolares se obtuvo información acerca de la profesión del jefe/a de familia y del nivel educacional del adulto a cargo, lo que permitió situarlos en el estrato medio-medio según la escala Graffar-Méndez Castellano (1994).

\section{Instrumentos}


Se seleccionaron dos instrumentos. Uno destinado a examinar el desempeño intelectual como criterio de exclusión. El otro para examinar las habilidades mentalistas.

\section{Evaluación de las habilidades mentalistas}

La Escala de Tareas de TM (Scaling of Theory-of-Mind Tasks) (Wellman y Liu, 2004), adaptada al español rioplatense (Romanazzi, 2014), incluye siete tareas que examinan secuencialmente las habilidades anteriormente mencionadas (1. Deseos diferentes, 2. Creencias diferentes, 3. Acceso al conocimiento, 4. Falsa creencia de contenido, 5. Falsa creencia explícita, 6. Creencia-emoción y 7. Emoción real-aparente). Para cada una de ellas se plantea una situación acompañada por un soporte visual, un objeto y/o una figura que representa a los personajes incluidos que ayudan a presentar y a recordar a los niños los contenidos y las opciones de respuesta ante las consignas que se les proporcionan (ver Anexo 1 sobre la Escala de la TM).

\section{Evaluación del desempeño intelectual}

Con el propósito de controlar la variable capacidad intelectual, además de las informaciones aportadas en los legajos escolares, se consideró el CI total obtenido a partir de la administración de la Escala de Inteligencia de Wechsler para preescolar y primaria (WPPSI III) (Wechsler, 2009), destinada a la evaluación de la inteligencia general en niños de 2 años 6 meses a 7 años 3 meses. Debido a la inexistencia de baremos locales, se utilizaron normas españolas para la puntuación de las respuestas obtenidas.

\section{Procedimiento y diseño}

Se trata de un estudio descriptivo transeccional (Hernández Sampieri, Fernández Collado y Baptista Lucio, 2010).

\section{Traducción y adaptación de la prueba}

Para la adaptación lingüística y cultural de las tareas de la escala (Romanazzi, 2014), se tuvo en cuenta el listado que proponen Hambleton y Zenisky (2011) para controlar la calidad de la traducción y la adaptación de ítems de evaluación. Estos autores confeccionaron dicho listado con el fin de facilitar la aplicación empírica de las directrices propuestas por la Comisión Internacional de Tests (Hambleton, 2001) para la adaptación de tests y cuestionarios.

Con el objetivo de lograr un nivel de dificultad y familiaridad del lenguaje de los ítems comparable con los del idioma original, se consultó a informantes clave (docentes y profesionales especialistas en infancia) mediante una entrevista no estructurada acerca de determinadas palabras, como sustantivos (particularmente nombres propios) y verbos, con las que se encuentran familiarizados los niños del medio local en las edades consideradas. Asimismo, se efectuó la desgrabación de conversaciones espontáneas llevadas a cabo en el ámbito de una institución educativa entre grupos de niños de la misma edad de los participantes y su maestra. Los nombres de los personajes de las tareas, los objetos que conformaron el material y algunos términos de las consignas fueron traducidos y adaptados sobre la base de la consulta y las desgrabaciones mencionadas. En la adaptación de la prueba se aseguró que los conceptos involucrados en los ítems resultantes de la adaptación se encontraran en el mismo nivel de abstracción que los de la versión original, que la longitud del enunciado y las alternativas de respuesta fueran similares y que no hubiera una modificación en la complejidad de la estructura gramatical, entre otras consideraciones.

\section{Obtención de datos}

En primer lugar, se gestionaron las autorizaciones institucionales y el consentimiento informado de los padres para la evaluación de los niños en los establecimientos educativos, 
garantizando la confidencialidad de los datos. Los niños fueron examinados individualmente fuera del aula, contemplando condiciones adecuadas de iluminación y sonido. La evaluación de cada niño estuvo a cargo de las autoras y se realizó en dos sesiones, de 40 minutos la primera y 20 minutos la segunda. Se procedió en primer lugar a administrar el WPPSI III, a partir de cuyos resultados se eliminaron los protocolos de niños que obtuvieron puntuaciones por debajo del CI total de 70. De este modo, de los 130 protocolos iniciales se excluyeron 25 . No se incluyeron niños de 3 años, dado que en el pilotaje no respondieron satisfactoriamente a ninguna de las tareas de TM.

\section{Análisis de datos}

Los resultados obtenidos en ambas pruebas fueron volcados a una base de datos y procesados estadísticamente.

Para el análisis de los resultados en torno al desempeño en las tareas de TM, en primer lugar, se adjudicó un puntaje de acuerdo a la respuesta correcta o incorrecta $(1$ y 0 , respectivamente). Se calcularon estadísticos descriptivos (frecuencias y porcentajes), destinados a comparar estos resultados con los de los autores de la escala. Se utilizaron estadísticos inferenciales (ANOVA) para evaluar el efecto de las variables género y edad sobre las habilidades mentalistas, y el test de Mc Nemar para identificar diferencias significativas de acuerdo al grado de complejidad entre pares de tareas.
Asimismo, se utilizó el programa Winsteps para realizar un análisis de la escala con el modelo de Rasch, para corroborar el grado de dificultad de los ítems (Prieto y Delgado, 2003).

Posteriormente, se recurrió a la aplicación del método de Guttman (Abdi, 2010) para determinar la escalabilidad de las mediciones. Esto es, para calcular en qué medida los patrones de respuesta de los sujetos incluidos se ajustan a los patrones de respuesta originales encontrados por los autores. Siguiendo el procedimiento propuesto por Guttman, se calcularon los coeficientes de reproductibilidad de cada ítem (CRi) y de cada sujeto (CRs). Dicho cálculo se realiza con el fin de detectar tanto aquellos ítems que podrían tener un mal funcionamiento como aquellos sujetos cuya actuación podría ser errática, de modo tal que la eliminación de tales casos contribuya a mejorar la escala.

\section{Resultados}

\section{Descripción del desempeño en la escala de TM}

Como se muestra en la Tabla 1, los porcentajes de respuestas correctas hallados en el grupo total son menores a los encontrados por Wellman y Liu (2004) teniendo en cuenta además, que no se incluyeron niños de 3 años. No obstante, cabe mencionar que solo en las tareas 5 y 7 , las diferencias resultaron estadísticamente significativas. 
Tabla 1

Porcentaje de niños que responden satisfactoriamente las pruebas mentalistas en el grupo local y en el estudio original.

\begin{tabular}{|c|c|c|c|c|c|}
\hline \multirow[b]{2}{*}{ Pruebas mentalistas } & \multicolumn{4}{|c|}{ Grupo local } & \multirow[b]{2}{*}{$\begin{array}{c}\text { Estudio original } \\
\text { 3-6 años (\%) } \\
\text { N }=75\end{array}$} \\
\hline & $\begin{array}{c}4 \text { años } \\
(\%) \\
N=45\end{array}$ & $\begin{array}{c}5 \text { años } \\
(\%) \\
N=38\end{array}$ & $\begin{array}{c}6 \text { años } \\
(\%) \\
N=22 \\
\end{array}$ & $\begin{array}{c}\text { Total } \\
\text { participantes } \\
(\%) \\
\mathbf{N}=\mathbf{1 0 5} \\
\end{array}$ & \\
\hline 1. Deseos diferentes & 89 & 86.8 & 95.5 & 89.5 & 95 \\
\hline 2. Creencias diferentes & 75.6 & 76.3 & 86.4 & 78.1 & 84 \\
\hline 3. Acceso al conocimiento & 46.7 & 63.2 & 100 & 63.8 & 73 \\
\hline 4. Falsa creencia de contenido & 35.6 & 55.3 & 86.4 & 53.3 & 59 \\
\hline 5. Falsa creencia explícita & 20 & 44.7 & 59.1 & 37.1 & $57^{*}$ \\
\hline 6. Creencia emoción & 20 & 42.1 & 81.8 & 41 & 52 \\
\hline 7. Emoción real aparente & 4.4 & 10.5 & 36.4 & 13.3 & $32 *$ \\
\hline
\end{tabular}

Por otra parte, los porcentajes de respuestas correctas disminuyeron de acuerdo a la complejidad de la tarea. Si bien en el grupo total el porcentaje de respuestas en las tareas de Falsa creencia explícita y Creencia-emoción, invierte el orden de dificultad encontrado por Wellman y Liu, aquí tampoco las diferencias alcanzaron significación estadística.

Del mismo modo que en los hallazgos reportados por los autores de la escala, el análisis de varianza (ANOVA) solo arrojó efectos significativos respecto de la edad, $[F(23,81)=3.17, p=.000]$ (Wellman y Liu, 2004 , p. 532). Dicho de otro modo, los porcentajes de respuestas acertadas aumentaron con la edad. No hubo efectos de género ni interacciones significativas.

\section{Dificultad de las tareas}

Complementariamente los datos fueron analizados con el modelo de Rasch. Este modelo suele utilizarse debido a que su carácter probabilístico resulta una ventaja frente a las limitaciones de la Teoría Clásica para la construcción y análisis de los tests. En la Tabla 2, puede observarse que el orden obtenido en este estudio respecto de la dificultad de los ítems de la escala, coincide con el propuesto por Wellman y Liu (2004). Convencionalmente, se suele situar la dificultad media de los ítems en el valor0, siendo los ítems con valores negativos los más fáciles de resolver (Prieto y Delgado, 2003). 
Tabla 2

Nivel de dificultad de los items según el modelo Rasch.

\begin{tabular}{c|c} 
Tareas & Nivel de dificultad del ítem \\
\hline 1. Deseos diferentes & -3.04 \\
2. Creencias diferentes & -1.79 \\
3. Acceso al conocimiento & -0.49 \\
4. Falsa creencia de contenidos & 0.11 \\
5. Falsa creencia explícita & 0.95 \\
6. Creencia emoción & 1.05 \\
7. Emoción real-aparente & 3.21
\end{tabular}

\section{Escalograma y comparación de los patrones}

Para establecer el orden de dificultad de los ítems de la escala, Wellman y Liu (2004) utilizaron el método del escalograma de Guttman (1944). Este modelo pretende la ordenación conjunta de personas y estímulos a partir de sus respectivos valores escalares. Habitualmente, el método se utiliza con un número reducido de ítems (de actitud, aptitud o rendimiento) que cumplen la función de estímulos y son formulados con niveles de dificultad creciente. El supuesto principal del modelo es que se pueden ordenar los ítems a lo largo de un continuo de forma tal que, si una persona responde adecuadamente a un ítem de determinado nivel de dificultad, debe hacer lo mismo con todos los ítems que estén por debajo de ese ítem en el continuo establecido. La escala de Guttman es unidimensional y acumulativa. Unidimensional porque los ítems corresponden a una sola dimensión subyacente y acumulativa porque ninguna persona tiene una respuesta positiva antes de una negativa.

Asimismo, bajo la consideración de que el método del escalograma resultaba rígido,
Wellman y Liu (2004) aplicaron a sus resultados el modelo de Rasch (1960) para ítems dicotómicos, que combina la dificultad del ítem y los niveles de habilidad de las personas. En su trabajo, tanto los niveles de dificultad de las tareas como los puntajes obtenidos por los examinados, son similares en ambos modelos de medición. No obstante, sostienen que "cualquier precisión ganada por el modelo de Rasch queda sopesada por la practicidad de puntuación de la Escala Guttman” (p. 536).

En ese mismo estudio, sobre siete de las tareas iniciales solo cinco se ajustaron al escalograma propuesto por Guttman. En las tareas 4 (Falsa creencia de contenidos), 5 (Falsa creencia explícita) y 6 (Creencia-emoción) obtuvieron niveles de dificultad similares. Por ese motivo, de acuerdo al criterio de Guttman incluyeron sólo una de ellas (Falsa creencia de contenidos). La escala finalmente quedó conformada por las tareas $1,2,3,4$ y 7 , para las que encontraron un coeficiente de reproductibilidad (CR) de .96 que se adecua a los valores requeridos ( $>$.90) (Abdi, 2010). A partir de esta escala, los autores derivaron seis patrones de respuesta (Tabla 3). 
Tabla 3

Patrones de respuesta de Wellman y Liu y del grupo local examinando: Frecuencia y porcentajes encontrados.

\begin{tabular}{|c|c|c|c|c|c|c|c|}
\hline Patrones de respuesta & 1 & 2 & 3 & 4 & 5 & 6 & Otros \\
\hline 1. Deseos diferentes & - & + & + & + & + & + & \\
\hline 2. Creencias diferentes & - & - & + & + & + & + & \\
\hline 3. Acceso al conocimiento & - & - & - & + & + & + & \\
\hline 4. Falsa creencia contenido & - & - & - & - & + & + & \\
\hline 7. Emoción real aparente & - & - & - & - & - & + & \\
\hline $\begin{array}{c}\text { n }(\%) \text { por patrón (Wellman } \\
\text { y Liu). } \mathrm{N}=75\end{array}$ & $1(1.33)$ & $4(5.33)$ & $11(14.66)$ & $8(10.66)$ & $19(25.33)$ & $17(22.6)$ & $15(20)$ \\
\hline $\begin{array}{l}\mathrm{n}(\%) \text { por patrón (Grupo } \\
\text { local) } \mathrm{N}=105\end{array}$ & $3(2.85)$ & $6(5.71)$ & $21(20)$ & $13(12.38)$ & $25(23.80)$ & $10(9.52)$ & $28(26.66)$ \\
\hline
\end{tabular}

Notas: Signo (-): falla en la tarea; signo (+): acierto en la tarea; Otros: distribución diferente en los aciertos.

Cuando se compararon los valores encontrados en los patrones de respuesta entre el grupo examinado y el informado por Wellman y Liu (Tabla 3), aparecieron diferencias de porcentaje que alcanzaron significación estadística en el patrón $6(\mathrm{Z}=2.435, p=.01)$, dando cuenta de un porcentaje inferior de respuestas correctas en el grupo local.

Teniendo en cuenta esa diferencia, para calcular en qué medida los patrones de respuesta de los participantes incluidos en este estudio se ajustaron a los patrones originales, se procedió en primer lugar, al análisis del escalograma conformado por la totalidad de los ítems, es decir, las siete tareas que componen la escala. Se encontró un coeficiente de reproductibilidad (CR) de .85 que no logró alcanzar los valores aceptables para considerarla una escala de alta capacidad predictiva.

Seguidamente, con el propósito de mejorar el escalamiento, se calcularon los coeficientes de reproductibilidad de cada ítem (CRi) y de cada sujeto (CRs).

Con respecto al $\mathrm{CRi}$, se encontró que ninguno de los ítems presentó un mal funcionamiento, siendo el valor del CRi mayor a la categoría modal en todos los casos. La categoría modal es la mayor de las proporciones de respuesta que presenta el ítem (se calcula el porcentaje de respuestas positivas $p$ y negativas $q$ que corresponden al ítem y se selecciona el mayor).

El cálculo del CRs permitió detectar seis casos que presentaron una actuación errática. Tales casos fueron entonces suprimidos con el objetivo de mejorar la escala. A continuación, se realizó nuevamente el cálculo del CR obteniendo un valor de .86 que, si bien implicó una mejora, no alcanza el valor esperable de .90 .

A continuación, se procedió al análisis de las tareas para seleccionar aquellas que mostraran un ajuste más adecuado a un escalograma. Se utilizó para ello el estadístico no paramétrico de Mc Nemar, que permite identificar diferencias significativas de acuerdo al grado de complejidad entre pares de tareas, tal como se observa en la Tabla 4. Se identificaron las dos tareas que resultaron semejantes en cuanto a su nivel de dificultad: las tareas 5 (Falsa creencia explícita) vs. 6 (Creencia-emoción), que hizo necesaria la elección de una de ellas.

Tabla 4 
Diferencias de complejidad entre pares de tareas.

\begin{tabular}{|c|c|c|}
\hline \multirow[b]{2}{*}{ Tareas comparadas } & \multicolumn{2}{|c|}{ Test de Mc Nemar } \\
\hline & $\begin{array}{c}\text { Resultados del } \\
\text { presente estudio } \\
\chi^{2}(1)[p]\end{array}$ & $\begin{array}{l}\text { Resultados } \\
\text { Wellman y Liu } \\
(2004) \chi^{2}(1)[p]\end{array}$ \\
\hline 1. 1. Deseos diferentes vs. 2. Creencias diferentes & $3.90(<.003)$ & $4.08(<.05)$ \\
\hline $\begin{array}{l}\text { 2. 2. Creencias diferentes vs. } 3 \text {. Acceso al } \\
\text { conocimiento }\end{array}$ & $6.56(<.01)$ & Sin datos \\
\hline $\begin{array}{l}\text { 2. 2. Creencias diferentes vs. } 4 \text {. Falsa creencia de } \\
\text { contenidos }\end{array}$ & $17.42(<.000)$ & $12.00(<.001)$ \\
\hline $\begin{array}{l}\text { 3. 3. Acceso al conocimiento vs. 4. Falsa creencia de } \\
\text { contenidos }\end{array}$ & $4.32(<.03)$ & $5.89(<.02)$ \\
\hline $\begin{array}{l}\text { 3. 3. Acceso al conocimiento vs. 5. Falsa creencia } \\
\text { explícita }\end{array}$ & $20(<.000)$ & Sin datos \\
\hline 4. 4. Falsa creencia contenidos vs. 6.Creencia emoción & $7.75(<.005)$ & Sin datos \\
\hline $\begin{array}{l}\text { 4.4. Falsa creencia contenidos vs. 5. Falsa creencia } \\
\text { explícita }\end{array}$ & $8.30(<.004)$ & Sin datos \\
\hline $\begin{array}{l}\text { 4. 4. Falsa creencia contenidos vs. 7. Emoción real } \\
\text { aparente }\end{array}$ & $38.20(<.000)$ & $13.88(<.001)$ \\
\hline 5. 5. Falsa creencia explícita vs. 6. Creencia emoción & $.024(<.877)$ & Sin datos \\
\hline $\begin{array}{l}\text { 5. 5. Falsa creencia explícita vs. 7. Emoción real } \\
\text { aparente }\end{array}$ & $13.08(<.000)$ & Sin datos \\
\hline 6. 6. Creencia emoción vs. 7.Emoción real aparente & $17.45(<.000)$ & Sin datos \\
\hline
\end{tabular}

Finalmente, se calculó el CR incluyendo dos grupos de seis tareas elegibles $(1,2,3,4$, 5 у 7, y 1, 2, 3, 4, 6 у 7). En ambos casos se encontró un CR de .88, no aceptable. Otras combinaciones probadas de cinco tareas $(1,2$, 3,5 y 7, y 1, 2, 3, 6 y 7) proporcionaron CR no apropiados: .89 y .88 , respectivamente.

Por consiguiente, se calculó el CR eliminando algunas de las tareas. En primer lugar, se consideraron las cinco tareas seleccionadas por Wellman y Liu (tareas 1, 2, 3, 4 y 7), para las que se obtuvo un CR de .90 , con un coeficiente de escalabilidad de .75 (son valores adecuados los mayores a .60). Aunque fue aceptable el CR de .90 de la escala que incluye los ítems 1, 2, 3, 4 y 7, una combinación de cuatro ítems (tareas 1, 2, 4 y 7) resultó la mejor opción del CR del escalograma, al mismo tiempo que obtuvo valores de significación estadística en los resultados del Mc Nemar. En este último caso se encontró un CR de .92 y un coeficiente de escalabilidad de .81 .

\section{Discusión y conclusiones}

En este trabajo se han presentado los resultados obtenidos con la Escala de TM de Wellman y Liu (2004) en niños preescolares que residen en la ciudad de La Plata, provincia de Buenos Aires (Argentina) y se los ha comparado con los informados por los autores en el estudio original.

$\mathrm{Su}$ interés reside, en primer lugar, en la significación que la capacidad mentalista tiene para el desarrollo psicológico infantil $\mathrm{y}$ en la escasez de estudios sobre el tema en 
el ámbito local. Al respecto los autores de la escala, siguiendo a Flavell (1972), destacanla importancia que tiene en los estudios evolutivos "el orden cronológico en el que las novedades cognitivas emergen durante la infancia" (p. 281). Asimismo, es de interés destacar la importancia de los estudios a nivel local, teniendo en cuenta lo señalado en distintas indagaciones sobre la diferencia de los resultados encontrados en diferentes países.

En segundo lugar, y en relación con el progreso en la comprensión conceptual de habilidades mentalistas en niños preescolares, el valor que reviste disponer de un método adecuado para evaluar con exactitud y de manera informativa el desarrollo sociocognitivo. Los datos proporcionados por Wellman y Liu ilustran acerca de la complejidad de las habilidades mentalistas y de su dominio progresivo con la edad. Se trata de una serie de adquisiciones conceptuales, progresivamente más elaboradas y flexibles que se constituyen a lo largo del desarrollo psicológico infantil, tal como se sostiene en otros trabajos (De Rosnay y Hughes, 2006; Dunn, 1988; Wellman, 1993). Las tareas incluidas conforman una escala a lo largo de un continuo, consistente con la interpretación sobre el desarrollo de la comprensión infantil de la TM durante los años preescolares, de modo tal que se cuenta con un instrumento adecuado para evaluar el progreso y expansión en su dominio.

En tercer lugar, cobran relevancia las posibles semejanzas o diferencias que podrían encontrarse en nuestro medio con respecto a la complejidad creciente de las tareas propuestas, a través de las cuales captar apropiadamente la secuencia del desarrollo de las habilidades mentalistas. Los resultados encontrados se inscriben en la misma línea de los autores y dan cuenta de dos cuestiones significativas. La primera alude a la adecuación de la adaptación de la escala. La segunda, a la confirmación de la necesidad de limitar las siete tareas originalmente propuestas a cinco, para ajustarse a los requerimientos de un escalograma en las edades consideradas. El CR de .90 y el coeficiente de escalabilidad de .75 son el resultado de la inclusión de las cinco tareas seleccionadas por Wellman y Liu (1, 2, 3, 4 y 7). Cabe señalar que para la opción de cuatro tareas $(1,2,4$ y 7) se encontró un CR de .92 y un coeficiente de escalabilidad de .81 más apropiados.

La escala resulta en consecuencia un instrumento adecuado, en la medida que proporciona evidencia empírica de la complejidad de las habilidades involucradas, así como de su progresión, imposibles de ser aprehendidas a través de una tarea única, tal como sucede en las evaluaciones que incluyen solo tareas de falsa creencia. Efectivamente, tanto los resultados de los creadores de la escala como los resultados de este estudio esclarecen el progreso en el dominio de la TM. Coincidentemente puede adherirse a la conceptualización según la cual la comprensión de deseos diferentes precede a la comprensión de creencias diferentes, la comprensión de creencias diferentes es anterior a la comprensión de falsa creencia y la diferenciación entre emociones reales y aparentes aparece más tardíamente en los años preescolares (Bartsch y Wellman, 1995; Wellman y Liu, 2004).

La adaptación de la escala utilizada permite la posible selección de las habilidades mentalistas a examinaren trabajos posteriores. Tanto la opción de cinco como de cuatro tareas, presentan un CR adecuado, siendo recomendable la de cinco, ya que posibilita una captación más amplia de las habilidades a evaluar.

De este trabajo se desprenden, además, dos observaciones sobre los resultados encontrados. El primero alude a que no fue posible incluir niños de 3 años, ya que en una exploración previa no respondieron satisfactoriamente a ninguna de las tareas, situación que contrasta con los hallazgos de Wellman y Liu en los que, de los 25 niños de esa edad, 15 proporcionaron distintos patrones de respuestas positivas, nueve patrones atípicos y solo uno no respondió acertadamente a ninguna.

El otro, vinculado al desempeño de los participantes incluidos, muestra diferencias 
significativas en el porcentaje de respuestas satisfactorias en el patrón 6: $22.66 \%$ en los resultados de Wellman y Liu y $9.52 \%$ en los del estudio que se informa. Alcanzan ese patrón: dos niños de 4 años, dos de 5 años y seis de 6 años. En el trabajo original, lo alcanzan cinco niños de 4 años y 12 de 5 años.

Estas diferencias respecto del estudio original no refieren al orden sino al tiempo de adquisición de las habilidades mentalistas, hallazgos que coinciden con estudios realizados en otros países (Liu, Wellman, Tardif y Sabbagh, 2008; Lu, Su y Whang, 2008; Wellman, Fang, Liu, Zhu y Liu, 2006; Wellman, 2016; Wellman, Cross y Watson, 2001). Tal como plantean Wellman et al. (2006), las comprensiones mentalistas de los niños reflejan una combinación de entendimientos (insights] universales y otros, culturalmente específicos. Si bien no es propósito de este trabajo explicar las diferencias encontradas, éstas podrían deberse al desempeño intelectual leventemente descendido de la muestra $(M=91.79)$.

Si bien se trata en ambos estudios de datos solamente descriptivos, su elucidación requiere de indagaciones más exhaustivas, con un mayor número de participantes de poblaciones no clínicas, en estudios no solo transeccionales sino también longitudinales y que incluyan correlaciones con otras variables como habilidad verbal, comprensión emocional, uso de palabras acerca de estados mentales entre madres e hijos, factores que han sido considerados predictores de la TM (como ejemplo véase Peterson y Slaughter, 2003).

Por otra parte, la escala resulta de utilidad para futuras indagaciones en poblaciones clínicas, que permitan esclarecer algunas de las diferencias ya constatadas en la literatura especializada, como es el caso de niños ubicados dentro del espectro autista y de niños con discapacidad intelectual, no siempre examinados con pruebas que varíen en su nivel y orden de dificultad. Asimismo, conviene señalar que en la actualidad el estudio de la
TM se extiende progresivamente hacia la consideración de competencias mentalistas avanzadas (comprensión de sinónimos, metáforas, ironías, lenguaje figurado) destinadas tanto a la consideración de la progresión de la TM como a la secuencia de su desarrollo en años posteriores, situación que conlleva la necesidad de profundizar el estudio sobre el tema en otras edades.

\section{Referencias bibliográficas}

Abdi, E. (2010). Guttman Scaling. En N. Salkind (Ed.), Encyclopedia of Research Design. Thousand Oaks, CA: Sage. Recuperado de https:// www.utdallas.edu/ herve/abdi-GuttmanScaling2010-pretty.pdf

Astington, J.W. (1998). El descubrimiento infantil de la mente. Madrid: Morata.

Astington, J.W. y Barriault, T. (2001). Children's theory of mind: how young children come to understand that people have thoughts and feelings. Infants \& Young Children, 13(3), 1-12. doi:10.1097/00001163-200113030-00005

Astington, J.W. y Jenkins, J.M. (1995). Theory of mind development and social understanding. Cognition and Emotion, 9, 151-165. http:// dx.doi.org/10.1080/02699939508409006

Romanazzi, J.(2014). Informe anual de Beca de Estudio. Comisión de Investigaciones Científicas de la Provincia de Buenos Aires. Recuperado de https://digital.cic.gba.gob.ar/ handle/11746/2818

Baron-Cohen, S., Leslie, A.M. yFrith, U. (1985). Does the autistic child have a "theory of mind"? Cognition, 21, 37-46. http://dx.doi. org/10.1016/0010-0277(85)90022-8

Bartsch, K.y Wellman, H.M. (1995). Children talk about the mind.New York: Oxford University Press.

Benavides Delgado, J. y Roncancio Moreno, M. (2009). Conceptos de desarrollo en estudios sobre Teoría de la Mente en las últimas tres décadas. Avances en Psicología Latinoamericana, 27(2), 297-310. Recuperado de http:// www.redalyc.org/articulo.oa?id=79915035007 Buitelaar, J.K., van der Wees, M., Swaab-Barne- 
veld, H. y van der Gaag, R.J. (1999). Theory of mind and emotion-recognition functioning in autistic spectrum disorders and in psychiatric control and normal children. Development and Psychopathology, 11, 39-58. http://dx.doi. org/10.1017/S0954579499001947

Carpendale, J. y Lewis, C.(2006).How children develop social understanding. (Understanding Children's Worlds). Malden: Blackwell Publishing.

Dennet, D. (1978). Beliefs about Beliefs. Behavioral \& Brain Sciences, 1(4), 568-570.

De Rosnay, M. y Hughes, C. (2006). Conversation and theory of mind: do children talk their way to socio-cognitive understanding? British Journal of Developmental Psychology, 24, 7-37. http:// dx.doi.org/10.1348/026151005X82901

Dunn, J. (1988). Sibling influences on childhood development. Journal of Child Psychology and Psychiatry, 29, 119-127. http://dx.doi. org/10.1111/j.1469-7610.1988.tb00697.x

Dunn, J., Brown, J., Slomkowski, C., Tesla, C. y Youngblade, L. (1991). Young Children's Understanding of Other People's Feelings and Beliefs: Individual Differences and Their Antecedents. Child Development, 62, 1352-1366. http://dx.doi.org/10.1111/j.1467-8624.1991. tb01610.x

Flavell, J.H. (1972). An analysis of cognitive-developmental sequences. Genetic Psychology Monograph, 86, 279-350.

Grafar Méndez-Castellano, H. y Méndez, M.C. (1994). Sociedad y estratificación, método Graffar Méndez Castellano. Caracas: Fundacredesa.

Guttman, L. (1944). A basis of scaling quantitative data. American Sociological Review, 9, 139-150. http://dx.doi.org/10.2307/2086306

Hambleton, R.K. (2001). The next generation of the ITC test translation and adaptation guidelines. European Journal of Psychological Assessment,17(3), 164-172. http://dx.doi. org/10.1027//1015-5759.17.3.164

Hambleton, R.K. y Zenisky, A.L. (2011). Translating and adapting tests for cross-cultural assessments. En D. Matsumoto y F.J.R. van de Vijver (Eds.), Cross-cultural research methods in psychology (pp. 46-70). Nueva York: Cambridge University Press. http://dx.doi. org/10.1017/CBO9780511779381.004

Harris, P.L., Donnelly, K., Guz, G.R. y PittWatson, R. (1986). Children's understanding of the distinction between real and apparent emotion. Child Development, 57, 895-909.

Harris, P.L., Johnson, C.N., Hutton, D., Andrews, G.y Cooke, T. (1989). Young children's theory of mind and emotion. Cognition \& Emotion, 3, 379-400.

Hernández Sampieri, M.C., Fernández Collado, C. y Baptista Lucio, P. (2010). Metodología de la investigación (5ta Ed.). México: McGraw Hill.

Kristen, S., Thoermer, C., Hofer, T., Aschersleben, G. y Sodian, B. (2006). Skalierung von Theory of Mind Aufgaben. Zeitschriftfür Entwick-ungspsychologie und Pädagogische Psychologie, 38, 186-195.

Liu, D., Wellman, H.M., Tardif, T. y Sabbagh, M.A. (2008). Theory of mind development in Chinese children: A meta-analysis of false-belief understanding across cultures and languages. Developmental Psychology, 44(2), 523-531. http://dx.doi.org/10.1037/00121649.44.2.523

Lu, H., Su, Y. y Wang, Q. (2008). Talking about others facilitates theory of mind in Chinese preschoolers. Developmental Psychology, 44(6), 1726-1736. http://dx.doi.org/10.1037/ a0013074

Milligan, K., Astington, J.W. y Dack, L.A. (2007) Language and Theory of Mind: Meta-analysis of the relation between language ability and false-belief understanding. Child Development, 78, 622-646. http://dx.doi.org/10.1111/ j.1467-8624.2007.01018.x

Olson, D. (1988). On the origins of beliefs and other intentional states in children. En J.W. Astington, P.L. Harris y D.R. Olson (Eds.), Developing theories of mind (pp. 414-426). Cambridge, UK: Cambridge University Press.

O'Reilly, K., Peterson, C.C. y Wellman, H.M. (2014). Sarcasm and advanced theory of mind understanding in children and adults with prelingual deafness. Developmental Psychology, 50(7), 1862-1877. http://dx.doi. 
org/10.1037/a0036654

Perner, J. (1988). Higher order beliefs and intentions in children's understanding of social interaction. En J.W. Astington, P.L. Harris y D.R. Olson (Eds.), Developing Theories of Mind (pp. 271-292). Cambridge, UK: Cambridge University Press.

Perner, J. (1994). Comprender la mente representacional. Barcelona: Paidós.

Perner, J., Leekam, S.R. y Wimmer, H. (1987). Three-year olds' difficulty with false belief. British Journal of Developmental Psychology, 5, 125-137. http://dx.doi.org/10.1111/j.2044835X.1987.tb01048.x

Peterson, C. y Slaughter, V. (2003). Opening windows into the mind: mothers' preferences for mental state explanations and children's theory of mind. Cognitive Development, 18(3), 399-429. http://dx.doi.org/10.1016/S08852014(03)00041-8

Peterson, C.C., Wellman, H.M. y Liu, D. (2005). Steps in theory of mind development for children with autism, deafness or typical development. Child Development, 76, 502-517.

Peterson, C.C., Wellman, H.M. y Slaughter, V. (2012), The Mind Behind the Message: Advancing Theory-of-Mind Scales for Typically Developing Children, and Those with Deafness, Autism, or Asperger Syndrome. Child Development, 83, 469-485. http://dx.doi. org/10.1111/j.1467-8624.2011.01728.x

Pillow, B.H. (1989). Early understanding of perception as a source of knowledge. Journal of Experimental Child Psychology, 47, 116-129. http://dx.doi.org/10.1016/00220965(89)90066-0

Pratt, C. y Bryant, P.E. (1990). Young children understand that looking leads to knowing (so long as they are looking into a single barrel). Child Development, 61, 973-982. http://dx.doi. org/10.2307/1130869

Premack, D. y Woodruff, G. (1978). ¿Tiene el chimpancé una teoría de la mente? En E. Martí (Comp.), Construir una mente (pp. 137-178). Barcelona: Paidós.

Prieto, G. y Delgado A.R. (2003). Análisis de un test mediante el modelo de Rasch. Psicothema,
15(1), 94-100.

Puche Navarro, R. (2002). Las Psicologías Cognitivas y la Psicología de la mente. Herencias, relaciones, tensiones y perspectivas. RevistaPensamientoPsicológico, 1, 25-46.

Rasch, G. (1960). Probabilistic models for some intelligence and attainment tests. Chicago: University of Chicago Press.

Resches, M., Serrat, E., Rostan, C. y Esteban, M. (2010). Lenguaje y Teoría de la Mente: una aproximación multidimensional. Infancia $y$ aprendizaje, 33(3), 315-333. http://dx.doi. org/10.1174/021037010792215136

Repacholi, B.M. y Gopnik, A. (1997). Early reasoning about desires: Evidence from 14- and 18-month-olds. Developmental Psychology, 33, 12-21. http://dx.doi.org/10.1037/00121649.33.1.12

Romanazzi, J. (2014). Informe anual de Beca de Estudio. Comisión de Investigaciones Científicas de la Provincia de Buenos Aires. Recuperado de https://digital.cic.gba.gob.ar/ handle/11746/2818

Serrano Ortiz, J. (2012).Desarrollo de la teoría de la mente, lenguaje y funcionesejecutivasenniños de 4 a 12 años (Tesis de doctorado inédita). Universitat de Girona. Departament de Psicología. Recuperado de http://hdl.handle. net/10803/123549

Siegal, M. y Beattie, K. (1991). Where to look first for children's understanding of false beliefs. Cognition, 38, 1-12.

Vesterinen, J. (2008). The TOM Storybooks as a tool of studying children's theory of mind in Finland (Tesis de doctorado inédita). Department of Psychology. University of Jyvaskyla. Finland.

Wellman, H.M. (1993). Early understanding of mind: The normal case. En S. Baron-Cohen, H. Tager-Flusberg y D.J. Cohen (Eds.), Understanding other minds: Perspectives from autism (pp. 10-39). Oxford, England: Oxford University Press.

Wellman, H. (1995). Desarrollo de la teoría del pensamiento en los niños. Bilbao: Desclée de Brouwer. 
Wellman, H. (2016). Cognición social y educación: Teoría de la Mente. Pensamiento Educativo. Revista de Investigación Educacional Latinoamericana, 53(1), 1-23. http://dx.doi. org/10.7764/PEL.53.1.2016.2

Wellman, H.M. y Bartsch, K. (1989). 3-year-olds understand belief. Cognition, 33, 321-326.

Wellman, H.M., Cross, D. y Watson, J. (2001). Meta-analysis of theory of mind development: The truth about false belief. Child Development, 72, 655-684. http://dx.doi.org/10.1111/14678624.00304

Wellman, H.M., Fang, F., Liu, D., Zhu, L. y Liu, G. (2006). Scaling of theory-of-mind understandings in Chinese children. Psychological Science,17, 1075-1081. http://dx.doi. org/10.1111/j.1467-9280.2006.01830.x

Wellman, H.M., Fuxi, F. y Peterson, C.C. (2011). Sequential Progressions in a Theory of Mind Scale: Longitudinal Perspectives. Child Development, 82(3), 780-792. http://dx.doi. org/10.1111/j.1467-8624.2011.01583.x

Wellman, H.M., Hollander, M. y Schult, C.A.
(1996). Young children's understanding of thought-bubbles and of thoughts. Child Development, 67, 768-788. http://dx.doi. org/10.2307/1131860

Wellman, H. y Liu, D. (2004). Scaling of Theory of Mind Tasks. Child Development, 75(2), 523-541. http://dx.doi.org/10.1111/j.14678624.2004.00691.x

Wellman, H.M. y Woolley, J.D. (1990). From simple desires to ordinary beliefs: The early development of everyday psychology. Cognition, 35, 245-275. http://dx.doi. org/10.1016/0010-0277(90)90024-E

Weschler, D. (2009). Test de Inteligencia para niños preescolares - WPPSI III. Madrid: TEA Ediciones.

Wimmer, H. y Perner, J. (1983). Beliefs about beliefs: representation and the constraining function of wrong beliefs in young children's understanding of deception. Cognition, 13, 103-128. http://dx.doi.org/10.1016/00100277(83)90004-5

Recibido: 5 de marzo de 2018 Aceptado: 9 de enero de 2020 


\section{Anexo 1}

\section{Escala de Teoría de la Mente de Wellman y Liu (2004)}

\section{(Traducción y adaptación: Romanazzi, 2014)}

\section{Instrucciones para su administración y puntuación}

El material incluye cajas, juguetes pequeños, títeres y hojas en las que están impresas distintas imágenes de objetos, así como los personajes que aparecen en las 7 tareas incluidas. Luego de proporcionar las instrucciones correspondientes a cada tarea, se formulan diferentes tipos de preguntas: preguntas de comprensión, preguntas objetivo y preguntas de control. Las respuestas a las preguntas se puntúan como correctas (1 punto) o incorrectas (0 puntos). El puntaje máximo es 7. A continuación se explicitan los objetivos, el material, el procedimiento de administración y los criterios de puntuación de cada una de las tareas seleccionadas.

\section{Tarea: Deseos diferentes ${ }^{2}$}

Objetivo: Diferenciar el deseo propio del ajeno sobre un mismo objeto.

Materiales: Una hoja con la imagen de un adulto y una hoja con las imágenes impresas de una zanahoria y una galletita.

Procedimiento: Se le muestra al niño la imagen del adulto y las imágenes de una zanahoria y una galletita. Luego se le dice: "Este es el Sr. Pérez. Es la hora de la merienda, por eso, el Sr. Pérez quiere algo para comer.

1) Pregunta sobre el deseo propio: Acá

hay dos comidas diferentes: una zanahoria y una galletita. ¿Cuál te gusta más?”.

Si el niño elige la zanahoria se le dice: "Bueno, esa es una buena elección, pero al Sr. Pérez le gustan mucho las galletitas. No le gustan las zanahorias. Lo que más le gusta son las galletitas" $(\mathrm{O}$, si el niño elige las galletitas, se le dice que al Sr. Pérez le gustan las zanahorias).

2) Luego se le hace la pregunta objetivo sobre el deseo ajeno: "Bueno, llegó la hora de comer. El Sr. Pérez sólo puede elegir una cosa para comer, sólo una. ¿Qué elegirá el Sr. Pérez? ¿Una zanahoria o una galletita?"

Puntuación: Para obtener 1 punto, el niño debe responder la pregunta objetivo de manera opuesta a su respuesta a la pregunta sobre su propio deseo.

\section{Tarea: Creencias diferentes ${ }^{3}$}

Objetivo: Discriminar creencias diferentes respecto de la propia sobre un mismo objeto, sin conocer cuál es verdadera o cuál es falsa.

Materiales: Una hoja con una imagen que representa a una niña y una hoja con las imágenes impresas de plantas y una cochera.

Procedimiento: Se le muestraal niño el dibujo de la niña y la hoja de papel con las imágenes de plantas y una cochera. Luego se le dice: "Acá está Lucía. Lucía quiere encontrar a su gato. Su gato podría estar escondido entre las plantas o en la cochera".

1) Se formula primero la pregunta sobre la creencia propia: “¿Dónde crees que está el gato? ¿En las plantas o en la cochera?”.

$\mathrm{Si}$ el niño elige las plantas se le dice: "Bueno, esa es una buena idea, pero Lucía cree que su gato está en la cochera". (O, si el niño elige la cochera, se le dice que Lucía cree que su gato está en las plantas).

2) Luego se le hace la pregunta objetivo sobre la creencia ajena: "Entonces,

\footnotetext{
${ }^{2}$ Tarea derivada de las utilizadas por Wellman y Woolley (1990) y Repacholi y Gopnik (1997) (Wellman y Liu (2004).

${ }^{3}$ Tarea derivada de las utilizadas por Wellman y Bartsch (1989) y Wellman et al. (1996) Wellman y Liu (2004).
} 
¿Dónde buscará Lucía a su gato? ¿En las plantas o en la cochera?".

Puntuación: Para obtener 1 punto, el niño debe contestar la pregunta sobre la creencia ajena de manera opuesta a su respuesta sobre su propia creencia.

\section{Tarea: Acceso al conocimiento ${ }^{4}$}

Objetivo: Predecir el conocimiento que puede tener el otro sobre un objeto.

Materiales: Un títere que representa a una niña y una caja de madera con tapa que contiene en su interior un auto de juguete.

Procedimiento: Se le muestra al niño la caja de madera con la tapa cerrada que contiene el en su interior al auto de juguete. Luego se le dice: "Acá hay una caja ¿Qué crees que hay dentro de la caja?” (El niño puede dar la respuesta que quiera o decir que no lo sabe). A continuación, se abre la caja y se le muestra al niño su contenido: "Vamos a ver... ¡Hay un auto adentro!”. Se cierra la tapa y se le pregunta: "Bueno, ¿qué hay en la caja?" (Respuesta esperada: "un auto").

Luego se le presenta el títere que representa a la niña y se le dice: "Esta es Lola que nunca vio qué hay dentro de la caja. Ahora acá viene Lola".

1) Se formula a continuación la pregunta objetivo sobre el conocimiento ajeno: "Entonces, ¿Lola sabe lo que hay en la caja?".

2) Luego se hace la pregunta de control que refiere a la memoria: "¿Lola vio adentro de la caja?".

Puntuación: Para obtener 1 punto, el niño debe responder a ambas preguntas de manera negativa (por ej. "Lola no sabe y Lola no vio qué había dentro de la caja").

\section{Tarea: Falsa creencia de contenidos 5}

Objetivo: Atribuir a otro una falsa creencia acerca del contenido de un recipiente prototípico una vez que se conoce el verdadero contenido de dicho recipiente, que no se corresponde con el del contenido usual.

Materiales: Un títere que representa a un niño y una caja de galletitas claramente identificable como tal con unos chanchitos de juguete en su interior.

Procedimiento: Se le muestra al niño la caja de galletitas cerrada que contiene en su interior los chanchitos de juguete (cuando se sacude el recipiente los objetos que están en su interior deberán hacer el mismo ruido que el que harían con el contenido usual -las galletitas). Luego se le dice: "Acá hay una caja de galletitas ¿Qué crees que hay adentro de la caja?". A continuación, se abre la caja y se dice: "Vamos a ver... ¡En realidad adentro hay unos chanchitos!". La caja se cierra. Luego se formula la pregunta que refiere a la memoria del niño: "Bueno, ¿Qué hay adentro de la caja de galletitas?" (Respuesta esperada: "chanchitos").

Luego se le muestra el títere que representa al niño y se le dice: "Este es Pedro y nunca vio qué había adentro de la caja de galletitas. Ahora acá viene Pedro".

1) Entonces, se formula la pregunta objetivo sobre el conocimiento: "¿Qué cree Pedro que hay en la caja? ¿Galletitas o chanchitos?'.

2) A continuación, se formula la pregunta de control que refiere a la memoria: "¿Pedro vio qué había adentro de la caja?".

Puntuación: Para obtener 1 punto, el niño debe contestar a la pregunta objetivo "galletitas" y negativamente a la pregunta control sobre memoria.

\footnotetext{
${ }^{4}$ Tarea derivada de las utilizadas por Pratt y Bryant (1990) y Pillow (1989), aunque con modificaciones para que su formato resultara similar al de la tarea de Falsa creencia (Wellman y Liu, 2004).

${ }^{5}$ Tarea derivada de las utilizadas inicialmente por Perner, Leekam y Wimmer (1987), ampliamente modificada y profusamente utilizada desde entonces (Wellman y Liu, 2004).
} 


\section{Tarea: Falsa creencia explícita ${ }^{6}$}

Objetivo: Reconocer que otro puede tener una creencia distinta de la realidad y por lo tanto falsa.

Materiales: Una hoja con la imagen de un niño y una hoja de papel con las imágenes impresas de una mochila y un armario.

Procedimiento: Se le muestra al niño la hoja con el dibujo que representa a un niño y la hoja con las imágenes impresas de una mochila y un armario. Luego se le dice: “Acá está Felipe. Felipe quiere encontrar sus guantes. Sus guantes podrían estar en su mochila o podrían estar en el armario. En realidad, los guantes de Felipe están en su mochila. Pero Felipe cree que sus guantes están en el armario".

1) Se formula la pregunta objetivo sobre la creencia del otro: "Entonces, ¿adónde va a ir Felipe a buscar sus guantes? ¿A su mochila o al armario?"

2) A continuación, se formula la pregunta sobre la realidad del lugar en el que se encuentran los guantes: "En realidad ¿dónde están los guantes de Felipe? ¿En su mochila o en el armario?".

Puntuación: Para obtener 1 punto, el niño debe responder a la pregunta objetivo: "al armario" y a la pregunta sobre la realidad de la ubicación de los guantes: "en la mochila".

\section{Tarea: Creencia-emoción ${ }^{7}$}

Objetivo: Predecir una emoción a partir de una creencia falsa.

Materiales: Un títere que representa a un niño y una caja de confites de tamaño real, claramente identificable, que contiene piedras adentro.

Procedimiento: Se le muestra al niño el títere que representa al niño y la caja de confites con las piedras adentro. Luego se le dice: "Acá hay una caja de confites y acá está Tomi. ¿Qué crees que hay adentro de la caja de confites?" (Respuesta esperada: "confites"). Luego el evaluador hace que Tomi hable: "Tomi dice: "Ay iqué bueno!, me encantan los confites. Los confites son mi golosina preferida. Ahora me voy a ir a jugar". Luego se aleja a Tomi y se lo deja fuera de la vista. A continuación, se abre la caja de confites y se le muestra al niño su contenido: “A ver... ¡En realidad hay piedras adentro de la caja, no confites! Sólo hay piedras". La caja de confites se cierra y se le pregunta: "Bueno, ¿cuál es la golosina preferida de Tomi?" (Respuesta esperada: "confites").

1) Se continúa con la pregunta objetivo sobre la emoción del otro: "Tomi vuelve y nunca vio qué había adentro de la caja y es la hora de la merienda. Vamos a darle a Tomi esta caja. Entonces, ¿cómo se siente Tomi cuando le dan la caja cerrada? ¿Contento o triste?".

2) Se prosigue con la pregunta control sobre la emoción del otro: El evaluador abre la caja de confites y deja que el juguete mire adentro: “¿Cómo se siente Tomi después de mirar adentro de la caja? ¿Contento o triste?".

Puntuación: Para obtener 1 punto, el niño debe responder a la pregunta objetivo sobre la emoción del otro "contento" y a la pregunta control de la emoción "triste".

\section{Tarea: Emoción real-aparente ${ }^{8}$}

Objetivo: Comprender que una persona puede sentir una determinada emoción, pero expresar una emoción diferente.

Materiales: Una hoja con la imagen de un niño de espaldas, de manera tal que la expresión facial no se puede ver y una hoja con las imágenes impresas de tres rostros que reflejan distintas emociones: felicidad, indiferencia y tristeza.

Procedimiento: Inicialmente, se le muestra al niño la hoja de papel con los tres

\footnotetext{
${ }^{6}$ Tarea derivada de las utilizadas por Wellman y Bartsch (1989) y Siegal y Beattie (1991) (Wellman y Liu, 2004).

${ }^{7}$ Tarea derivada de la utilizada por Harris, Johnson, Hutton, Andrews y Cooke (1989) (Wellman y Liu, 2004).

${ }^{8}$ Tarea derivada de la utilizada por Harris, Donnelly, Guz y Pitt-Watson (1986) (Wellman y Liu, 2004).
} 
rostros - feliz, neutral y triste- para constatar su conocimiento de esas expresiones emocionales preguntándole “¿Cómo está este nene?” con respecto a cada una de ellas. Luego el papel se pone a un lado y la tarea comienza mostrándole la figura que representa al niño de espaldas. Se le dice: "Esta es la historia de un nene. Voy a preguntarte sobre cómo realmente se siente el nene y cómo lo expresa en su cara. En realidad, el nene podría sentirse de una manera, pero mostrar algo diferente en su cara. O él podría sentirse de una forma y mostrar lo mismo en su cara. Quiero que me digas cómo se siente realmente y como se ve su cara. Se trata de la historia de Mati. Los amigos de Mati estaban jugando y contando chistes. Una de las nenas más grandes, Romi, hizo un chiste feo sobre Mati y todos se rieron. A todos les pareció muy gracioso excepto a Mati. Pero Mati no quería que los otros nenes vieran cómo se sentía por el chiste, porque pensó que ellos lo tratarían como si fuera un bebé. Por eso Mati intentó esconder lo que sentía".

Luego se hacen dos preguntas para controlar la memoria del niño sobre el relato: “¿Qué hicieron los otros nenes cuando Romi dijo un chiste feo sobre Mati?" (Respuesta esperada: "se rieron o pensaron que era divertido"). Se formula a continuación la otra pregunta de control sobre la memoria del niño: "En la historia que te conté ¿Qué habrían hecho los otros nenes si se hubiesen enterado de cómo se sentía Mati?". (Respuesta esperada: “Tratarlo como un bebé o burlarse de él").

1) Se pasa luego a la pregunta objetivo sobre sentimiento real: Señalando a las 3 imágenes que representan distintas emociones se le pregunta: "Entonces, ¿Cómo se sintió realmente Mati cuando todos se rieron? ¿Se sintió feliz, triste o normal?"

2) A continuación, se formula la pregunta objetivo sobre la apariencia: "¿Cómo trató Mati de que se viera su cara cuando todos se rieron? ¿Se veía feliz, triste o normal?".

Puntuación: Para obtener 1 punto, la respuesta del niño a la pregunta objetivo sobre sentimiento real debe ser más negativa que su respuesta a la pregunta objetivo sobre apariencia (por ej.: triste para sentimiento real y feliz o normal para sentimiento aparente, o normal para sentimiento real y feliz para sentimiento aparente). 
